\title{
Radial focusing and energy compression of a laser-produced proton beam by a synchronous rf field
}

\author{
Masahiro Ikegami, ${ }^{1}$ Shu Nakamura, ${ }^{2, *}$ Yoshihisa Iwashita, ${ }^{1}$ Toshiyuki Shirai,${ }^{1}$ Hikaru Souda, ${ }^{1}$ Yujiro Tajima, ${ }^{1}$ \\ Mikio Tanabe, ${ }^{1}$ Hiromu Tongu, ${ }^{1}$ Hiroyuki Itoh, ${ }^{1}$ Hiroki Shintaku, ${ }^{1}$ Atsushi Yamazaki, ${ }^{1}$ Hiroyuki Daido, ${ }^{2}$ Akifumi Yogo, ${ }^{2}$ \\ Satoshi Orimo, ${ }^{2}$ Michiaki Mori, ${ }^{2}$ Mamiko Nishiuchi, ${ }^{2}$ Koichi Ogura, ${ }^{2}$ Akito Sagisaka, ${ }^{2}$ Alexander S. Pirozhkov, ${ }^{2}$ \\ Hiromitsu Kiriyama, ${ }^{2}$ Shyuhei Kanazawa, ${ }^{2}$ Shuji Kondo, ${ }^{2}$ Yoichi Yamamoto, ${ }^{2}$ Takuya Shimomura, ${ }^{2}$ Manabu Tanoue, ${ }^{2}$ \\ Yoshimoto Nakai, ${ }^{2}$ Atsushi Akutsu, ${ }^{2}$ Sergei V. Bulanov, ${ }^{2}$ Toyoaki Kimura, ${ }^{2}$ Yuji Oishi, ${ }^{3}$ Koshichi Nemoto, ${ }^{3}$ \\ Toshiki Tajima, ${ }^{2}$ and Akira Noda ${ }^{1}$ \\ ${ }^{1}$ Institute for Chemical Research, Kyoto University, Gokasho, Uji, Kyoto, 611-0011, Japan \\ ${ }^{2}$ Advanced Photon Research Center and Photo Medical Research Center, Japan Atomic Energy Agency, 8-1-7 Umemi-dai, \\ Kizu, Kyoto 619-0215, Japan \\ ${ }^{3}$ Central Research Institute of Electric Power Industry, 2-6-1 Nagasaka, Yokosuka, Kanagawa 240-0196, Japan
}

(Received 15 June 2007; revised manuscript received 15 October 2008; published 30 June 2009)

The dynamics of a $\mathrm{MeV}$ laser-produced proton beam affected by a radio frequency (rf) electric field has been studied. The proton beam was emitted normal to the rear surface of a thin polyimide target irradiated with an ultrashort pulsed laser with a power density of $4 \times 10^{18} \mathrm{~W} / \mathrm{cm}^{2}$. The energy spread was compressed to less than $11 \%$ at the full width at half maximum (FWHM) by an rf field. Focusing and defocusing effects of the transverse direction were also observed. These effects were analyzed and reproduced by Monte Carlo simulations. The simulation results show that the transversely focused protons had a broad continuous spectrum, while the peaks in the proton spectrum were defocused. Based on this new information, we propose that elimination of the continuous energy component of laser-produced protons is possible by utilizing a focal length difference between the continuous spectral protons and the protons included in the spectral peak.

DOI: $10.1103 /$ PhysRevSTAB.12.063501

PACS numbers: 41.85.-p, 41.75.Jv, 52.38.Kd

\section{INTRODUCTION}

An increase in the acceleration gradient of chargedparticle beams has long been required for fundamental researches, such as particle and nuclear physics, in order to obtain high-energy particles within a limited length of the accelerators. From the point of view of such applications as hadron therapy and material treatment, the downsizing of ion accelerators by using a high acceleration gradient is also important. The emission of energetic ions from a plasma created by a high-intensity short-pulse laser has been reported [1,2]. Recent experiments have shown that a multi-MeV ion beam was stably generated from a laser-irradiated thin foil [3-12]. Laser-plasma ion acceleration is one of the candidates of a compact accelerator [13]. For actual use, monochromatization of the broad energy spectrum should be developed. Recently, several approaches to create quasimonoenergetic ion beams have been tested in several laboratories [14-17] which, however, seems to be difficult for such an operation with a moderate repetition rate (up to $10 \mathrm{~Hz}$, for example) with good reproducibility. In order to produce monoenergetic ion beams with high repetition-rate operation, we have pro-

\footnotetext{
*Present address: High Energy Accelerator Research Organization (KEK), 1-1 Oho, Tsukuba, Ibaraki 305-0801, Japan.
}

posed the combination of laser-plasma acceleration with a phase-rotation method by a synchronous if electric field [18-21].

Phase rotation is a method in which the configuration of the particle distribution in longitudinal phase space is rotated by an rf electric field so as to realize a small energy spread. Laser-produced ions are emitted within a very short time duration $(\sim 1 \mathrm{ps})$ from the plasma created by an ultrashort laser pulse with a duration shorter than $100 \mathrm{fs}$. Since the ions have a variety of energy, after the passage of a certain drift distance ( $\sim 1 \mathrm{~m}$ in our experiment), ions with higher energy arrive at the gap of the rf cavity earlier, and those with a lower energy arrive later. If the phase of the $\mathrm{rf}$ field is adjusted so that the earlier arrived ions are decelerated and the ions arriving later are accelerated, we can collect the ions to a certain energy region.

In the present work, the dynamics of laser-produced proton beams (with a broad energy spectrum) was studied by applying an $\mathrm{rf}$ electric field in both experiments and (computer situations) calculations.

\section{EXPERIMENTAL SETUP}

The present experiment was carried out at the Kansai Photon Science Institute, Japan Atomic Energy Agency, using a Ti:sapphire laser system, called J-KAREN [22], with the setup shown in Fig. 1. In this experiment, the peak 


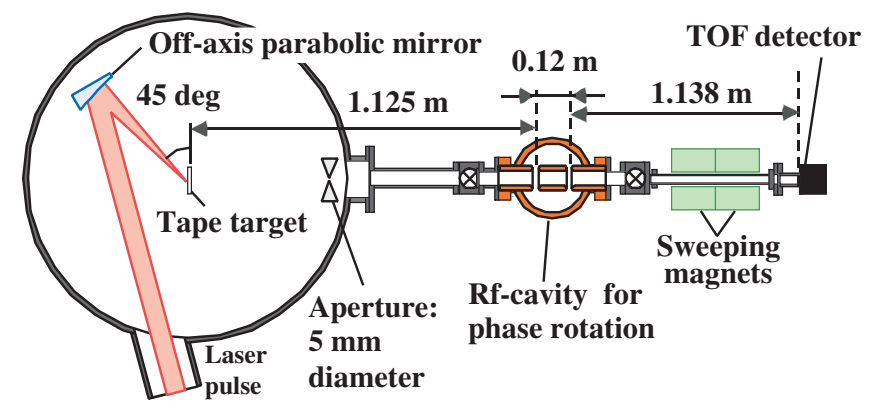

FIG. 1. (Color) Schematic view of the experimental setup of phase rotation. The protons emitted normal to the rear surface of the tape target are injected into the rf cavity. The flight time of protons with and without phase rotation is measured by a TOF detector. The sweeping magnets are used to remove electrons so as to suppress the background in the scintillation counter of the TOF detector.

power and the pulse duration were up to $18 \mathrm{TW}$ and $25 \mathrm{fs}$, respectively [23]. The repetition rate of the laser was $10 \mathrm{~Hz}$. The laser pulse was focused by an off-axis parabolic mirror with a focal length of $325 \mathrm{~mm}$, and the focused energy was $0.45 \mathrm{~J}$ on a $7.5 \mu \mathrm{m}$-thick polyimide tape target giving an intensity of $4 \times 10^{18} \mathrm{~W} / \mathrm{cm}^{2}$ on the target. The laser pulse was incident on the target surface with an angle of 45 degrees, as shown in Fig. 1. The tape target was rolled up so as to be irradiated on a fresh surface during continuous irradiation. The protons were emitted normal to the rear surface of the target. The emission time of the protons was estimated to be less than $\sim 1$ ps. The protons which were emitted from the target had a Gaussian distribution in the transverse direction $[24,25]$, and their divergence solid angle to the target normal was $3.8 \times 10^{-1}$ sr in $2 \sigma$, where $\sigma$ represents the standard deviation. The protons passed through an aperture with a $5 \mathrm{~mm}$ diameter before injection to a phase-rotation rf cavity. The solid angle of the aperture was $9.1 \times 10^{-5}$ sr. In this small solid angle, the radial distribution of the protons was almost uniform. If the protons produced at the target had the above-mentioned divergence solid angle $\left(3.8 \times 10^{-1} \mathrm{sr}\right)$, about $0.4 \%$ of them could pass through the aperture. At the rf cavity, the faster protons were decelerated and the slower protons accelerated. In order to develop a time spread of the beam required for phase rotation, the protons must pass through a certain distance. Therefore, the rf cavity was set at $1.125 \mathrm{~m}$ downstream from the target (Fig. 1). The rf cavity had a hole to pass through a beam of which the inner diameter was $50 \mathrm{~mm}$. The solid angle of this hole was larger than the $5 \mathrm{~mm}$ diameter aperture. Therefore, all of the protons that passed through the aperture could be accepted.

The rf cavity is composed of a quarter-wave length resonator. The cavity has two gaps. The gap size and the voltage amplitude are $20 \mathrm{~mm}$ and $80 \mathrm{kV}$, respectively. The frequency of the $\mathrm{rf}$ is $82 \mathrm{MHz}$. To measure the rf phase and the gap voltage, the rf signal is monitored through the pickup port of the rf cavity. The rf voltage applied to the cavity is synchronized with the pulsed laser so that the rf electric field could be applied with a fixed phase relation to the pulsed laser in order to efficiently compress the energy spread of the laser-produced protons.

Seed pulses to enter into Ti:sapphire amplifiers are generated by a mode-locked oscillator having a frequency of $82 \mathrm{MHz}$. Among these $82 \mathrm{MHz}$ pulse trains, laser pulses are selected at a $10 \mathrm{~Hz}$ repetition rate, and then further amplified. When we use an $82 \mathrm{MHz}$ oscillator of the seed pulses as the master oscillator for the rf, the phase relation between the time of laser irradiation to the target which is the time of proton pulse duration and the rf phase will be well defined. Note that laser pulse width as well as proton pulse width is almost negligibly short compared with the period of rf. The source signal of the rf from the master oscillator is picked up by a PIN photodiode and amplified by three-stage rf amplifiers up to $30 \mathrm{~kW}$ and fed to the rf cavity. Although only one cycle of the rf electric field is sufficient for the purpose of an energy compression of a pulsed beam generated by each laser pulse, rf power is fed with the duration of up to $\sim 1 \mathrm{~ms}$ ( $\sim 10^{5}$ periods) into the cavity to realize stable operation. This rather long duration of rf power feeding is also necessary to cover the filling time to build up a sufficient rf voltage (stored energy) in the cavity before the arrival of protons. The timing jitter between the laser pulse and the rf electric field is negligible. This rather long pulsed operation of the rf cavity (duty factor of up to $1 \%$ ) contributes to the reduction of the power dissipation and heat generation at the cavity wall to a tolerable level.

\section{ENERGY SPECTRUM}

The energy of the protons is measured with a time-offlight (TOF) detector [26,27]. The solid angle of the TOF detector is $1.7 \times 10^{-4}$ steradian (sr) to the tape target in the setup shown in Fig. 1. This is larger than the solid angle of the $5 \mathrm{~mm}$ diameter aperture. Therefore, all of the protons that passed through the aperture could be detected in the present experiment. The time-of-flight signal of protons measured by the TOF detector is shown in Figs. 2(a) and 2(b) by solid lines. The vertical axis corresponds to the number of protons that entered into the aperture of the detector. In Fig. 2(a), the solid gray line is the TOF spectrum of laser-produced protons averaged over ten shots in the case without an rf field. The black solid line is the TOF spectrum averaged over ten shots when the rf field for phase rotation is applied. It is found that the distribution of the TOF signals is strongly modulated by phase rotation. In Fig. 2(b), each solid line represents a spectrum shot by shot. The structure of the TOF distribution is reproduced precisely for every laser shot. In the absence of an rf field, the horizontal axis can easily be converted to the energy of a proton, because proton velocity is constant during the flight from the target to the detector. The energy spectrum converted from the mea- 


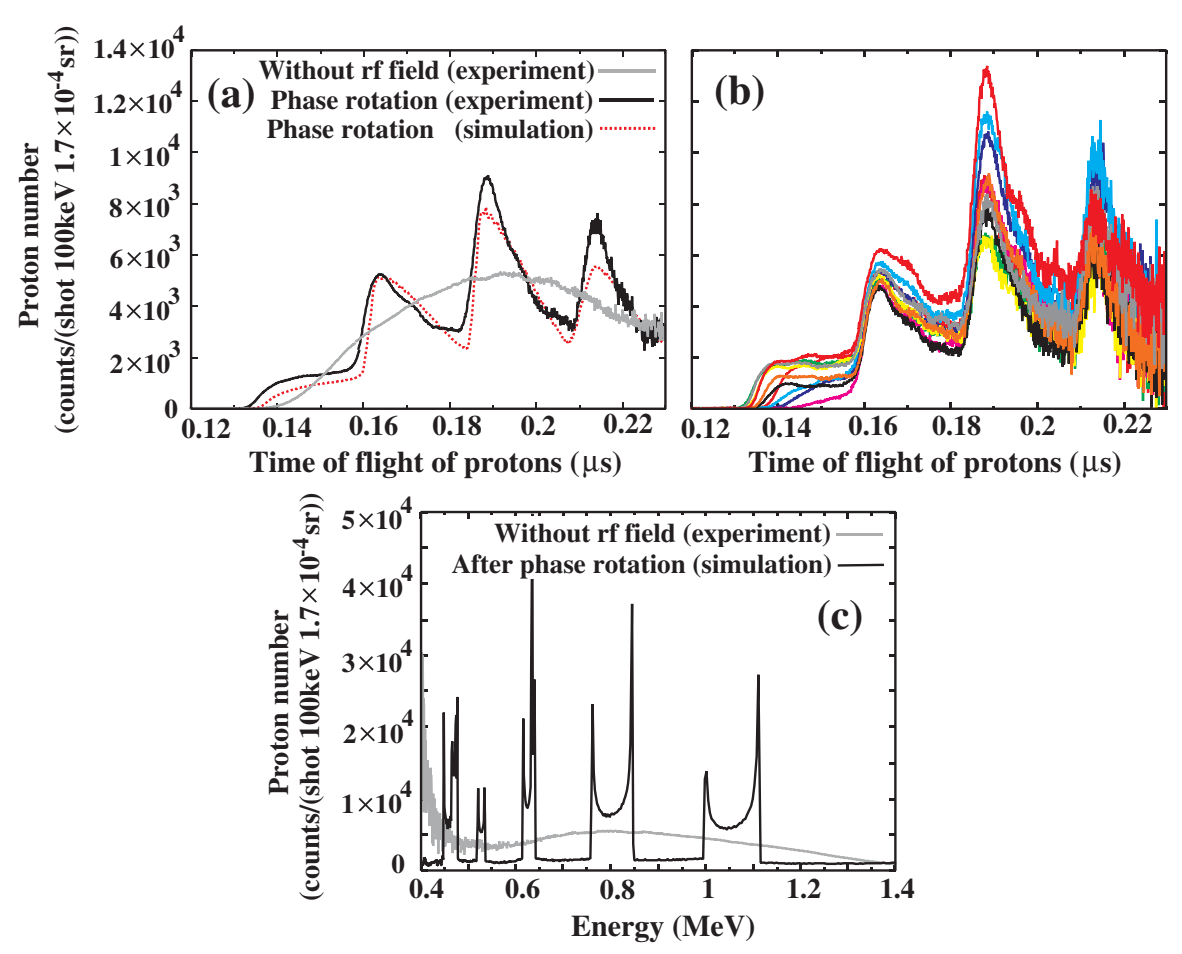

FIG. 2. (Color) (a) TOF signal with and without the phase rotation. The horizontal axis is the arrival time of protons from the foil target to the TOF detector. Part (b) shows the TOF signal shot by shot. Although the number of protons is different shot by shot, the global structure of the distribution is reproduced precisely. (c) Energy spectra corresponding to the TOF spectra of (a).

sured TOF signal is shown by the gray solid line in Fig. 2(c). When the protons are accelerated or decelerated by the rf cavity, the velocities change during the flight. Therefore, in the case with phase rotation, the TOF does not directly give the correct proton energy.

In order to obtain the exact kinetic energy of the phaserotated protons, we should calculate the velocity change at the rf gap. Those protons having the energy spectrum of the gray solid line of Fig. 2(c) are assumed to pass through the rf field of the cavity. The voltage, frequency, and phase of the cavity are set at the same condition as in the experiment. Then, from the calculation, the energy spectrum after the phase rotation is evaluated, as shown in Fig. 2(c) by the black solid line. The energy spread of the spectral peak at $1.05 \mathrm{MeV}$ is $11 \%$ at FWHM (including two partial peaks). In this peak, $10^{4}$ protons are estimated to be included. These correspond to $0.07 \%$ of protons produced at the target. Calculation of the flight time to the TOF detector with use of the simulated proton energy spectrum gives us a TOF spectrum similar to the experimental one, as shown by the dotted line in Fig. 2(a). This result shows the relevance of our calculation. It should be noted that the TOF method smooths out the fine structure of the original energy distributions because it cannot take into account the velocity change at the rf cavity. Therefore, in order to obtain the exact energy spectrum of the phase-rotated beam, we should calculate the energy spectrum from the observed TOF spectrum by the above procedure. The energy spectrum after phase rotation includes several spec- tral peaks, as shown in Fig. 2(c). The proton intensity increases by more than a factor of 2 in each peak after phase rotation. Each peak can be separated by an analyzing magnet put downstream of the cavity. Then, we obtain a quasimonoenergetic proton beam.

In the following, we quantitatively show the effect of the phase rotation on the energy spectrum. The interval of each peak in the energy spectrum is decided by the rf frequency and the distance between a cavity and a target, as follows. The energy gain of a charged particle that passes through the rf gap can be written as

$$
\Delta E=q V T \cos \left(\phi+\phi_{0}\right)
$$

where $q, V$, and $T$ are the charge, voltage amplitude, and transit time factor, respectively [28]. The notation $\phi$ is the phase of the $\mathrm{rf}$ when a proton reaches the $\mathrm{rf}$ gap which is given by $\phi=2 \pi f L / v$, where $f, L$, and $v$ are the frequency of the rf, the distance from the target to the rf gap, and the velocity of protons, respectively. The notation $\phi_{0}$ is the initial rf phase. For simplicity, we consider that the rf initial phase, $\phi_{0}$, is set at $0.5 \pi$. Then, protons that arrive at the gap within the phase $n \pi<\phi<(n+1) \pi$ gain energy (accelerated), and those within $(n+1) \pi<\phi<(n+2) \pi$ lose energy (decelerated), where $n$ is a positive integer. As a result, protons are collected to velocities of

$$
v_{n}=\frac{f L}{n}, \quad n=1,2 \ldots
$$

each of which is the central velocity of the $n$th peak created 
in the energy spectrum. In the present experiment, the energy peak at $1.05 \mathrm{MeV}$ corresponds to the 6th peak. The phases of adjacent peaks should satisfy the relation $\phi_{n+1}-\phi_{n}=2 \pi f L / v_{n+1}-2 \pi f L / v_{n}=2 \pi$, where $\phi_{n}$ is the rf phase corresponding to the $n$th peak. The velocity difference, $\Delta v=v_{n}-v_{n+1}$, of adjacent peaks indicates the velocity range collected to the $n$th spectral peak. The acceptable velocity range of the $n$th spectral peak is written as

$$
\frac{\Delta v}{v_{n}}=\frac{v_{n}}{v_{n}+f L} .
$$

Therefore, if the $\mathrm{rf}$ frequency $f$ or distance from the target to the $\mathrm{rf}$ gap $L$ is reduced, the velocity range collected to a peak is increased.

\section{TRANSVERSE FOCUSING AND DEFOCUSING}

Control of the transverse profile of the protons accelerated in a laser plasma is described in Ref. [16] based on a destructive method that is not suitable for repetitive operation. Generally, it is known whether charged particles that pass through an rf gap receive a transverse kick, depending on the rf phase [28]. In our experiment, we found that the radial component of the rf electric field clearly gives focus or defocus effects on the motion of the protons in the transverse direction. The transverse profile of the proton beam is detected at 1.736 and $2.365 \mathrm{~m}$ downstream of the tape target (the proton source) by using a nuclear track detector, CR-39 [29]. Since CR-39 detection has sensitivity only to heavy particles (protons, ions, and neutral atoms), it is suitable for proton detection under heavy backgrounds of laser light, laser-generated hard $\mathrm{x}$ rays, and electrons. The surface of the CR-39 is covered with a thin foil so that only specific protons higher than a certain threshold energy are detected. By changing the thickness of the foil carefully, we can choose the energy range of the detected protons. In the present experiment, the thickness of the foil is adjusted so that protons higher than $0.78 \mathrm{MeV}$ also can be detected. The cross section of the proton beam detected by the CR39 is shown in the upper pictures of Figs. 3(a)-3(c). When we do not apply phase rotation, the radial distribution of protons measured at $1.736 \mathrm{~m}$ downstream from the tape target becomes as shown in Fig. 3(a). It is found that the divergence angle of the proton beam is $5.4 \mathrm{mrad}$, which is determined by the diameter of the aperture in Fig. 1. In the cross section of the beam, the proton distribution is almost uniform. When the rf field for phase rotation is switched on, the radial distribution drastically changed as shown in Fig. 3(b). This is detected at the same position as shown in Fig. 3(a). Both focusing and defocusing components are visible. When the phase-rotated beam is observed further downstream $(2.365 \mathrm{~m})$ from the tape target, the inner component becomes small and dense. Therefore, we could judge that this component is focusing. On the other hand, the outer components are defocused. It is a matter of
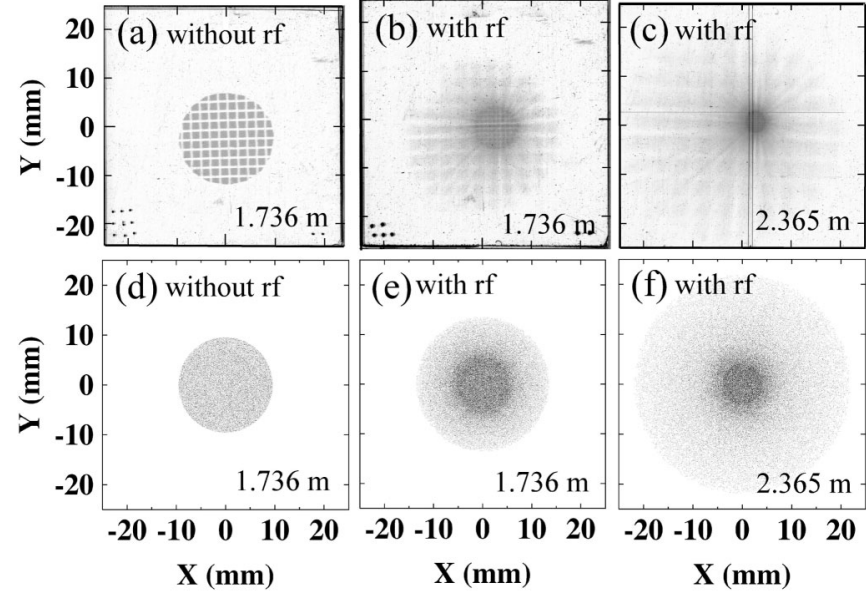

(f) with rf

FIG. 3. Comparison of the transverse profile of the beam. (a)(c) (upper pictures) are the profile detected in the experiment. (d)-(f) (lower pictures) are the profile obtained by Monte Carlo simulation with the same condition to the experiment. Parts (a), (b), (d), and (e) are distributions at $1.736 \mathrm{~m}$ downstream from the tape target the proton source. Parts (a) and (d) are the profile without phase rotation, (b) and (e) are with phase rotation. Parts (c) and (f) are measured at $2.365 \mathrm{~m}$ downstream from the tape target with phase rotation. In the experiment a mesh was inserted between the target and the rf cavity to measure the divergence. From (c), a diameter of the defocusing component is estimated to be about $40 \mathrm{~mm}$ at $2.365 \mathrm{~m}$ downstream from the target. The TOF detector is put at $2.383 \mathrm{~m}$ downstream from the target, and it has an aperture of $42 \mathrm{~mm}$. Therefore, a significant part of the focused protons was detected by the TOF detector, even when the rf cavity was switched on in the present experiment.

concern whether the protons that have a specified energy are selectively focused, or not. In the present measurement we could not distinguish the energy between the focusing and defocusing components, while the threshold of the energy could be identified. Identification of the energy of the focused and defocused protons gives important information for understanding the dynamics of phase-rotated beams.

In order to clarify such a problem, we carry out a Monte Carlo simulation assuming a time-dependent rf field for phase rotation. Protons are tracked in six-dimensional phase space with the method of transfer matrix [30]. By using this simulation, we could identify the energy of protons that have various radial positions. In order to evaluate the justification of the simulation, we first compared the transverse projection of protons, under the same condition as in the experiment. A radially uniform proton beam having the energy spectrum as shown by the solid gray line in Fig. 2(c) was assumed to pass through the rf gap with a divergence angle of $5.4 \mathrm{mrad}$. As shown in the lower pictures of Figs. 3(d)-3(f), the simulation results are qualitatively consistent with the experimental results.

By using this simulation method, we can discuss the relation between the radial distribution and the energy spectrum. The transverse projection of protons of the en- 
ergy between 0.85 and $0.99 \mathrm{MeV}$, which corresponds to the valley of the energy spectrum, was investigated by the simulation. As shown in Fig. 4(b), in this energy region, only the focusing component is included. The radial distribution of protons between an energy of 0.99 and $1.11 \mathrm{MeV}$, which corresponds to the peak of the energy spectrum, is shown in Fig. 4(c). The distribution has two spatial components: one is defocused, and the other seems to be more focused. For the other energy peaks, similar results were obtained. This means that the focused protons
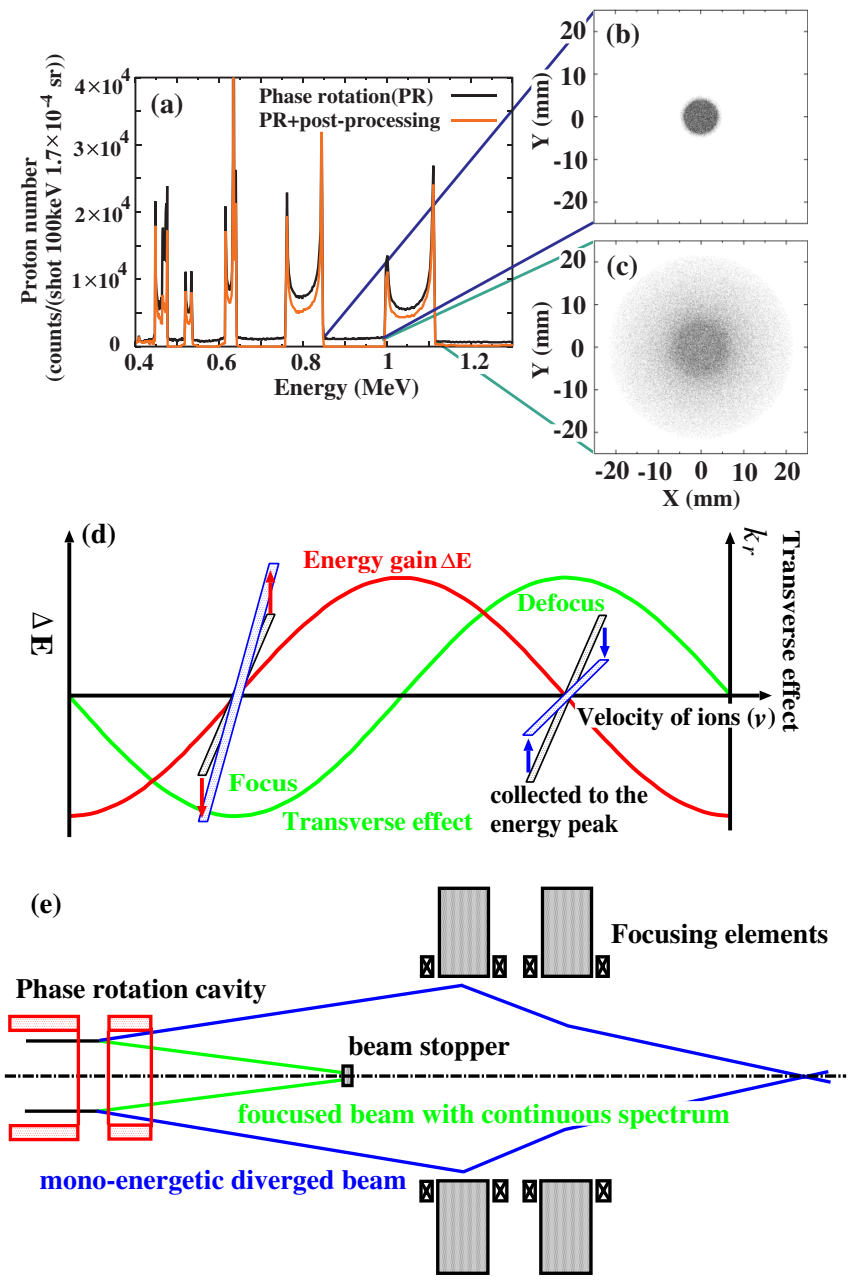

FIG. 4. (Color) (a) Simulated energy spectra of phase-rotated beam. The transverse profile is also simulated by the Monte Carlo method. Parts (b) and (c) show the simulated transverse profile of phase-rotated proton beam included in the valley of the energy spectrum (between 0.85 and $0.99 \mathrm{MeV}$ ) and that in the energy peak (between 0.99 and $1.11 \mathrm{MeV}$ ). Parts (b) and (c) are calculated at $2.365 \mathrm{~m}$ downstream of the tape target (the proton source) with the same condition as Fig. 3. Part (d) is a schematic illustration of the relation between the strength of the effects of energy compression and transverse focusing. The transverse axis is written with the velocity. (e) Setup of postprocessing used to remove protons with a continuous spectrum. If the phase-rotated beam passes through such a system, the energy spectrum is expected to change as shown in (a). are distributed over the whole energy spectrum, while the defocused protons are collected in the energy spectral peak. Such a property of a phase-rotated beam can be explained as follows. The focusing strength in the transverse direction $\left(k_{r}\right)$ (this corresponds to the reciprocal of the focal length) is given by the following formula [30]:

$$
k_{r}=\frac{-\pi q V T \sin \left(\phi+\phi_{0}\right)}{m v^{2} \gamma^{2} \lambda}
$$

where $m, v$, and $\gamma$ are the mass, velocity, and Lorentz factor of the proton, respectively. $T$ is the transit time factor used in Eq. (1). $\lambda$ is the wavelength of the rf. $\phi$ is the phase of the rf which is identical to that of Eq. (1); $\phi=2 \pi f L / v$. The positive sign of $k_{r}$ means a defocusing effect, and the negative sign of $k_{r}$ means focusing. We consider Eqs. (1) and (4) as being a function of velocity, $v$. Then, for the present experimental parameters, the trigonometric functions (cosine and sine) are dominant for the determination of the signs and absolute values of $\Delta E$ and $k_{r}$ in the velocity region of the present laser-produced protons $(\sim 1.5 \mathrm{MeV})$. In Fig. 4(d), we illustrate the transverse [ $k_{r}$; Eq. (4)] and longitudinal [ $\Delta E$; Eq. (3)] effects as functions of the velocity of a proton over a period of the rf. In this figure, the transverse effect of the phase rotation can be divided into focusing and defocusing phases. Since the laser-produced protons arrive in the phase-rotation cavity with a variety of velocities, the protons are injected into all of the phases. We consider the protons that have velocities included in the right half of Fig. 4(d) (this velocity region corresponds to a transverse defocusing phase). Since these protons also have an energy spread, their distribution can be illustrated as the parallelogram shown in the $v-\Delta E$ space of Fig. 4(d). In this region the faster protons lose energy and the slower ones gain energy. As a result, the protons at the transversely defocusing phase are compressed in energy and a spectral peak is created. On the other hand, the protons that have velocities corresponding to the left half of Fig. 4(d) are focused in the transverse direction. In this region the faster protons gain energy and the slower protons lose energy. This means that the total proton energy spread increases. The net result is that after the phase rotation the protons belonging to the continuous part of the energy spectrum have been radially focused, while those in the spectral peaks defocused. The results of Figs. 3(b), 3(c), 3(e), and 3(f) and of Figs. 4(b) and 4(c) can be explained by this interpretation.

If we utilize such a property of phase-rotated protons and ions, we should cut the tail of each quasimonoenergetic peak in the energy spectrum. After phase rotation, focused protons (ions) always have a continuous energy spectrum. Fortunately, these protons (ions) are focused within a small radius near the focal point, as shown in Figs. 4(b) and 4(c). Therefore, if the focused components are removed by a beam stopper at the focal point, those protons (ions) with a continuous energy spectrum can be 
removed. Utilizing this feature by the setup shown in Fig. 4(e), we can select only the energy peaks. In order to evaluate the efficiency of this method, a Monte Carlo simulation assuming the same conditions as the previous simulation was performed. A beam stopper of $5 \mathrm{~mm}$ in radius was placed at $1.5 \mathrm{~m}$ downstream of the cavity to remove the focused component. The distance of $1.5 \mathrm{~m}$ corresponds to the focal length of the focusing component. According to the simulation, the intensity of the spectral peak is slightly reduced after this postprocessing. On the other hand, the protons that are included in the valley of the energy spectrum are completely removed [Fig. 4(a)].

\section{CONCLUSIONS}

We successfully created quasimonoenergetic peaks in the energy spectrum of $\mathrm{MeV}$ proton beams by combining laser-plasma acceleration and energy modulation by using a phase-rotation cavity. We found that $0.07 \%$ of protons produced at the target were collected to the highest quasimonoenergetic peak. This method has a capability to create monoenergetic beams with a relatively high repetition rate. We also showed that the phase-rotation changes the radial distribution of laser-produced protons, depending on their position in the energy spectrum. The protons included in the energy peak were defocused, and the almost focused protons belonged to a continuous energy spectrum. Utilizing the difference in the focal length between the quasimonoenergetic protons and those having a continuous energy spectrum, we could remove the continuous energy component. A series of postprocessing for the laserproduced protons shown in this paper can open up the feasibility of improving quality and reproducibility of the laser-produced proton and ion beams for practical applications [31].

\section{ACKNOWLEDGMENTS}

This work was partly supported by "Advanced Compact Accelerator Development Project" which belongs to the Ministry of Education, Culture, Sports, Science and Technology (MEXT) of Japanese government, and it was also partly supported by the Grant-in-Aid for the Global COE Program "The Next Generation of Physics, Spun from Universality and Emergence" of the MEXT of Japan. This work was also partly supported by the
Special Coordination Fund (SCF) for Promoting Science and Technology commissioned by the MEXT of Japan.

[1] A. P. Fews et al., Phys. Rev. Lett. 73, 1801 (1994).

[2] F. N. Beg et al., Phys. Plasmas 4, 447 (1997).

[3] E. L. Clark et al., Phys. Rev. Lett. 84, 670 (2000).

[4] S. P. Hatchett et al., Phys. Plasmas 7, 2076 (2000).

[5] A. Maksimchuk, Phys. Rev. Lett. 84, 4108 (2000).

[6] Y. Murakami et al., Phys. Plasmas 8, 4138 (2001).

[7] K. Krushelnick et al., IEEE Trans. Plasma Sci. 28, 1184 (2000).

[8] K. Nemoto et al., Appl. Phys. Lett. 78, 595 (2001).

[9] H. Schwoerer, P. Gibbon, S. Düsterer, R. Behrens, C. Ziener, C. Reich, and R. Sauerbrey, Phys. Rev. Lett. 86, 2317 (2001).

[10] K. Matsukado et al., Phys. Rev. Lett. 91, 215001 (2003).

[11] A. Fukumi et al., Phys. Plasmas 12, 100701 (2005).

[12] M. Nishiuchi et al., Phys. Plasmas 15, 053104 (2008).

[13] D. Habs, G. Pretzler, A. Pukhov, and J. Meyer-ter-vehn, Prog. Part. Nucl. Phys. 46, 375 (2001).

[14] B. M. Hegelich et al., Nature (London) 439, 441 (2006).

[15] H. Schwoerer et al., Nature (London) 439, 445 (2006).

[16] T. Toncian et al., Science 312, 410 (2006).

[17] S. Tar-Avetisyan et al., Phys. Rev. Lett. 96, 145006 (2006).

[18] A. Noda et al., in Proceedings of the 7th Linear Accelerator Conference, LINAC 2002, Vienna (EPS, Geneva, 2002), p. 644.

[19] A. Noda et al., Laser Phys. 16, 647 (2006).

[20] S. Nakamura et al., Jpn. J. Appl. Phys. 46, L717 (2007).

[21] A. Noda et al., Int. J. Mod. Phys. B 21, 319 (2007).

[22] H. Kiriyama et al., Opt. Lett. 33645 (2008).

[23] M. Mori et al., IEEE Trans. Plasma Sci. 36, 1872 (2008).

[24] A. Yogo et al., Phys. Rev. E 77, 016401 (2008).

[25] M. Nishiuchi et al., Appl. Phys. Lett. 94, 061107 (2009).

[26] S. Nakamura et al., Jpn. J. Appl. Phys. 45, L913 (2006).

[27] A. Yogo et al., Phys. Plasmas 14, 043104 (2007).

[28] S. Y. Lee, Accelerator Physics (World Scientific, Singapore, 1999), p. 374.

[29] A. P. Fews and D. L. Henshaw, Nucl. Instrum. Methods Phys. Res. 197, 517 (1982); A. P. Fews, Nucl. Instrum. Methods Phys. Res., Sect. B 71, 465 (1992).

[30] K. R. Crandall and D.P. Rusthoi, Los Alamos National Laboratory Report No. LA-UR-90-4146, p. 17.

[31] U. Linz and J. Alonso, Phys. Rev. ST Accel. Beams 10, 094801 (2007). 